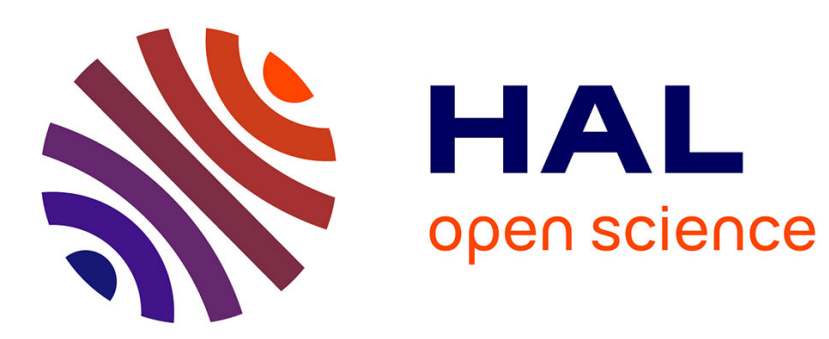

\title{
Communication et domination sociale en Franconie à la fin du Moyen Âge: l'enjeu de la réponse
}

\author{
Joseph Morsel
}

\section{To cite this version:}

Joseph Morsel. Communication et domination sociale en Franconie à la fin du Moyen Âge: l'enjeu de la réponse. 2005. halshs-00388554

\section{HAL Id: halshs-00388554 \\ https://shs.hal.science/halshs-00388554}

Preprint submitted on 26 May 2009

HAL is a multi-disciplinary open access archive for the deposit and dissemination of scientific research documents, whether they are published or not. The documents may come from teaching and research institutions in France or abroad, or from public or private research centers.
L'archive ouverte pluridisciplinaire HAL, est destinée au dépôt et à la diffusion de documents scientifiques de niveau recherche, publiés ou non, émanant des établissements d'enseignement et de recherche français ou étrangers, des laboratoires publics ou privés. 


\title{
Communication et domination sociale en Franconie à la fin du Moyen Âge : l'enjeu de la réponse
}

\author{
Joseph Morsel \\ Université Panthéon-Sorbonne (Paris I) / Institut Universitaire de France
}

\begin{abstract}
Cette boite [ $=$ le poste de radio] parlait sans s'arrêter et ne semblait pas s'intéresser aux réponses; cela lui valut rapidement le nom de As'oromagb'esi [= Celui qui parle sans attendre les réponses]. Une nouvelle ligne fut alors ajoutée à la ritournelle née au moment de l'arrivée de l'électricité, et plus tard, cette ritournelle rendit aussi hommage à Lagos, où le monopole sacré du parasol, réservé jusque-là à la royauté, avait été brisé pour la première fois: Elektiriki ina oba / Umbrella el'eko / As'oromagb'esi, iro oyinbo [= Électricité, lumière du gouvernement / Parasol, pour l'élite de Lagos / 'Celui qui parle sans attendre les réponses', mensonge de l'homme blanc].

(Wole Soyinka, Ake, the Years of Childhood, 1981)
\end{abstract}

«L'espace public au Moyen Âge » ? Le premier commentaire que j'ai griffonné sur la lettre d'appel à participation que Patrick Boucheron et Nicolas Offenstadt nous ont envoyée a été le suivant : «L'espace public est un espace de domination que nous qualifions de 'public' ». Au-delà du caractère radical, en tout cas nonhabermassien de cette annotation, on voit surtout que ce qui m'avait interpellé, c'est l'usage de la notion de «public», à propos duquel je n'ai jamais cessé, par écrit ou devant les étudiants, comme d'autres d'ailleurs, de dénier toute pertinence à l'importation de notre distinction courante privé/public dans la société médiévale'. Ce faisant, j’acceptais tacitement la notion d'« espace », qui pourtant n’a pas plus de légitimité en soi que le second segment. On est en effet en droit de s'interroger sur la pertinence et surtout sur les effets intellectuels de la traduction d’Öffentlichkeit par « espace public».

\section{I.-LÖffentlichkeit, un espace public?}

Chez Habermas, l'Öffentlichkeit s'incarne certes dans des lieux publics (cafés, clubs et salons) mais il ne s'y réduit pas et, sauf erreur de ma part, l'espace n'est pas un dimension-clé de l'Öffentlichkeit' : l'espace n'est intégré que de manière implicite et latérale à sa théorie, sous la forme des lieux où se constitue l'Öffentlichkeit, et il me semble qu'il faut attendre longtemps avant qu'Habermas n'utilise la notion d'öffentlicher Raum (p. ex. en 2004, dans son discours de remerciement pour le Prix de Kyoto). Conséquemment, l'Öffentlichkeit a des lieux, elle n'est pas un espace. D'ailleurs, la consultation des versions étrangères accessibles par Internet montre que seule la traduction française fait clairement référence à l'espace : le norvégien opte pour un terme transparent eu égard à sa proximité linguistique de l'allemand (offentlighet), l'italien et l'espagnol choisissent «opinion publique (opinione pubblica, opinión pública), l'anglais et le néerlandais parlent de «sphère publique » (public sphere, publieke sfeer) - qu'on pourrait certes créditer d'une certaine connotation spatiale, mais outre que dans les deux cas le syntagme «espace public » était possible (public space, publieke ruimte), sphere et sfeer sont des notions plus abstraites, évoquant métaphoriquement un

\footnotetext{
${ }^{1}$ Jürgen Habermas, Strukturwandel der Öffentlichkeit. Untersuchungen zu einer Kategorie der bürgerlichen Gesellschaft, (1962) 2e éd revue, Neuwied/Berlin, Luchterhand (Politica, 4), 1965, p.15, la rejette lui aussi d'emblée (" es hat einen Gegensatz zwischen Öffentlichkeit und Privatsphäre nach antikem (oder modernem) Modell nicht gegeben »), en s'appuyant notamment sur Otto Brunner, Land und Herrschaft (1959), qui voit dans la familia médiévale une fusion du privé et du public, tandis que publicus signifie plutôt, en suivant les historiens du droit, « commun » (gemein). L'espace public serait alors à trouver soit dans la familia, soit dans la communauté - ce qui n'a sans doute pas joué un rôle mineur dans le fait que les études actuelles outre-Rhin sur l'Öffentlichkeit médiévale adoptent massivement un cadre communautaire.

2. Habermas, Strukturwandel..., p. 11 : «'Öffentlich' nennen wir Veranstaltungen, wenn sie, im Gegensatz zu geschlossenen Gesellschaften, allen zugänglich sind - so wie wir von öffentlichen Plätzen sprechen oder von öffentlichen Häusern. » À travers cette analogie, Habermas semble donc admettre la possibilité d'une spatialité öffentlich - bien que la mention de «wir» ne renvoie pas clairement à lui-même, car Habermas dit en général «i ich » : il se référerait donc plutôt à l'usage commun. Mais surtout il poursuit : «Aber schon die Rede von 'öffentlichen Gebäuden' meint nicht nur deren allgemeine Zugänglichkeit ; sie müssen nicht einmal für den öffentlichen Verkehr freigegeben sein; sie beherbergen einfach Einrichtungen des Staates und sind als solche öffentlich. Der Staat ist die 'öffentliche Gewalt'. Er verdankt das Attribut der Öffentlichkeit seiner Aufgabe, für das öffentliche, das gemeinsame Wohl aller Rechtsgenossen zu sorgen. » L'éventuelle spatialité d'öffentlich n'est donc qu'accidentelle et seconde.
} 
milieu/domaine/champ social ${ }^{3}$. Et dans tous les cas, les notions de «sphère publique » (Sphäre der Öffentlichkeit) et d'« opinion publique » (öffentliche Meinung) étaient chez Habermas.

Tout semble donc indiquer que la spatialité de l'Öffentlichkeit est une dimension introduite par la traduction en français - dont le lexique se caractérise justement par une importante et courante spatialisation, sur laquelle Gérard Genette avait attiré l'attention dès les années 1960. Le problème n'est cependant pas de dénoncer une quelconque infidélité notionnelle à une pure thèse habermassienne, mais d'attirer l'attention sur les risques intellectuels de l'opération consistant à importer sans crier gare un mode de lecture non assumé et non construit. Car autant Öffentlichkeit est, en allemand, d'emblée une notion abstraite ("publicité » en tant que le fait d'être public/ouvert/accessible), autant en français «espace public » entraîne avec soi la référence concrète à des lieux publics, à une étendue tridimensionnelle susceptible d'être investie par des pratiques cérémonielles et/ou discursives, à des lieux de rencontre plus ou moins institués (cours, églises, cimetières, marchés, etc.). Bref, la notion d'« espace public» me semble impliquer la conception substantialiste de l'espace qui est au cœur de nos représentations courantes ${ }^{4}$, raison pour laquelle Jean-Philippe Genet avait pu s'insurger lors de la première rencontre contre la «réification» de l'espace public à laquelle il lui semblait assister'5.

L'Öffentlichkeit est ainsi moins un lieu de communication qu'un rapport de communication, qui peut certes s'instaurer dans des lieux spécifiques (p. ex. les cafés, clubs et salons de Habermas), mais qui n'est pas lié à ces lieux. Et c'est parce que l'Öffentlichkeit constitue moins un espace de communication qu'un rapport de communication qu'il importe de s'efforcer de l'appréhender à travers les rapports de communication. Ceux-ci sont configurés à la fois par les positions et postures sociales des partenaires de la communication et par les effets sociaux spécifiques des médias employés. C'est pourquoi, en guise de contribution à la compréhension des structures de la communication médiévale et, au-delà, à la discussion de la notion d'espace public médiéval, je partirai de la présentation de deux pratiques instituées de communication concernant un espace et un temps homogènes (la Franconie à la fin du Moyen Âge) : l'organisation conceptuelle du champ scriptural et l'aveu de droits. Celles-ci posent le problème de la domination sociale à travers les pratiques de communication, ce qui signifie que j’aborderai le problème de l'Öffentlichkeit médiévale à l'envers, à partir de ce que Habermas appellerait la «féodalisation » de l'Öffentlichkeit... Bref, je ne partirai pas de l'examen de lieux ou pratiques dont je pourrais penser a priori que l'Öffentlichkeit s'y trouve, mais de la communication dominante.

\footnotetext{
3. Chez Habermas, Sphäre (équivalent à Bereich, cf. la substitution notionnelle Intimsphäre/Intimbereich p. 39-40) n'a pas non plus une signification spécifiquement spatiale : elle désigne une forme sociale qui certes $a$ un espace, mais n'est pas un espace. Il peut ainsi dire que la maison est « der innere Bezirk der Privatsphäre » (p. 39).

4. Sur cette conception substantialiste dominante de l'espace, notamment chez les historiens de toutes périodes, cf. en première approche J. MORSEL, «Appropriation communautaire du territoire, ou appropriation territoriale de la communauté ? Observations en guise de conclusion ", dans: Hypothèses 2005. Travaux de l'École doctorale d'histoire de l'Université Paris I Panthéon-Sorbonne, Paris, Publications de la Sorbonne, à paraitre (2006). L'absence de spatialité dans l'approche de Habermas et notre tendance à une lecture spatialisante de la métaphore d'« espace publique » s'explique sans doute assez aisément dès lors que l'on examine, même rapidement, l'évolution de la place de la spatialité dans la conception du lien social en Occident. La place (!) me manque ici pour le faire en détail et je me contenterai donc de schématiser : on doit d'une part constater la mise à l'écart du spatial dans l'étude du social qui a sous-tendu la formation de la pensée sociologique occidentale, en particulier chez Weber et Durkheim (les deux principales figures tutélaires), quoique de deux manières différentes (Durkheim réduit l'espace au substrat matériel des actes sociaux et renvoie l'étude particulière de ce substrat, réduit à du naturel, à la géographie, tout autant victime de la formation du champ sociologique que l'histoire. Quant à Weber et aux wébériens, ils n'accordent qu'une importance minime à la spatialité des phénomènes sociaux dans l'étude des modes de sociation : l'espace est éliminé au cours du processus d'abstraction analytique). Cette déspatialisation de l'analyse sociale est notamment au cœur de l'analyse des réseaux, qu'elle soit menée par les sociologues ou les historiens. L'approche de Habermas est de ce point de vue strictement conforme aux représentations scientifiques du social qui ont prévalu durant l'essentiel du XXe siècle. Mais à partir de la fin des années 1980 et surtout depuis les années 1990 se produit, dans les sciences sociales (sociologie, ethnologie, géographie, histoire...) un phénomène que l'on a pris l'habitude de désigner, à la suite d'Edward Soja (Postmodern Geographies : The Reassertion of Space in Critical Social Theory, Londres, Verso, 1989), comme spatial turn (en français «tournant géographique », en raison de l'intérêt que lui a porté prioritairement la géographie en France : cf. Le Débat, 92, 1996). Ce «tournant spatial» se traduit par la multiplication effrénée de travaux et publications sur l'espace ou la spatialité (cf. le rapide survol dans ma contribution mentionnée ci-dessus, n. 1), mais au-delà de ce qui pourrait n'apparaître que comme un effet de mode, il a surtout pour effet de nous sensibiliser à la spatialité, de convoquer la spatialité dans notre appréhension des phénomènes sociaux (présents ou passés) - et de spatialiser notre lecture de travaux qui pouvaient bien, selon la remarque de $G$. Genette, ne faire de l'espace qu'un usage métaphorique... Le risque est donc grand de lire de manière substantialiste des concepts qui n'étaient que métaphoriques!

5. Cette spatialité importée explique précisément qu'il a pu être évoqué à plusieurs reprises au cours de la seconde journée des entreprises de « déterritorialisation » de l'analyse de l'Öffentlichkeit, p. ex. celles d'Hélène Merlin, c'est-à-dire en fait d'analyses non spatialisées dont on nous a justement dit qu'il s'agissait d'entreprises parmi les plus conséquentes de prise en compte de la théorie habermassienne.
} 
À l'arrière-plan de cette approche se trouve l'idée que l'éventuelle «féodalisation » de l'Öffentlichkeit ne serait pas une situation en soi, parce que médiévale, le degré zéro de l'Öffentlichkeit à partir duquel tout se serait construit, mais qu'il ne peut s'agir que d'un produit historique. C'est par l'examen complet des rapports de communication médiévaux (ce que n'avait pas fait Habermas, qui s'est contenté d'utiliser, classiquement, le Moyen Âge comme repoussoir/contre-modèle des Lumières) que la pertinence notionnelle de l'Öffentlichkeit pourra être précisée, à la fois dans l'absolu et pour le Moyen Âge. Bref, je prendrai au mot Patrick Boucheron, qui avait évoqué lors de la rencontre introductive le devoir qui nous incombe de validation des théories à l'aune de résultats empiriques : il ne s'agit en l'occurrence pas de savoir s'il y avait ou non une Öffentlichkeit au Moyen Âge ${ }^{6}$, mais plutôt de voir si la notion est heuristiquement fructueuse ou non pour comprendre la société médiévale et, réciproquement, de voir en quoi la situation du Moyen Âge pourrait permettre de perfectionner la notion...

Je partirai de la manière dont la taxinomie médiévale franconienne structure de façon fondamentalement binaire le champ lexical de la communication écrite et formulerai une hypothèse susceptible d'en rendre compte. Les résultats obtenus seront alors confrontés à ceux de l'examen d'un rapport de communication spécifique, la Weisung, qui est a priori strictement contradictoire avec l'Öffentlichkeit de Habermas puisqu'elle est une forme de domination seigneuriale sur les dépendants qui y prennent part. Ceci me permettra de souligner une dimension particulière des rapports de communication dont la prise en compte est, d'une part, tout à fait compatible avec la démarche de Habermas mais, d'autre part, permet d'articuler autrement le binôme critique/adhésion qui structure l'évolution historique habermassienne.

\section{II.-Brief et schrift}

L'examen des pratiques documentaires franconiennes, visant non pas à la description de celles-ci mais à en faire apparaitre les régularités, a été mené à deux niveaux : non pas celui de la production de l'écrit, sur laquelle on sait toujours trop peu de choses (pourquoi écrit-on au Moyen Âge ?), mais la manière d'en parler et la manière de le conserver - qui déterminent largement notre approche des sources médiévales.

\section{1) Une terminologie bipolaire}

Lorsqu'on examine, à partir d'un corpus de sources franconiennes constitué aléatoirement entre la fin du XIII et le début du XVIe siècle ${ }^{7}$, le champ lexical des termes allemands qui désignent les documents écrits, on remarque immédiatement la domination écrasante de deux termes, brief et schrift : ils couvrent $98 \%$ de l'ensemble des occurrences, avec une très forte domination de brief (75,5\% des occurrences) sur schrift $(22,5 \%)$. Les deux termes couvrent donc l'essentiel du champ de ce que nous appelons « document écrit » - mais du document écrit non relié, car ces mots ne servent jamais à désigner un livre ou livret, pour lequel existent des mots exclusifs (c'est-à-dire non utilisés pour des documents volants) : liber, buch (ou büchlein), register, manual, etc. - une terminologie mal étudiée et sur laquelle je ne m’attarderai pas ici. Je soulignerai simplement le fondement visiblement matériel/technique de la distinction entre 'livre' et brief ou schrift, comme une sorte de prolongement de la distinction entre codex et pagina.

\footnotetext{
6. La même chose vaut évidemment aussi pour toutes les notions propres à notre société, p. ex. l'individu, la famille, le marché, etc. dont seul, au mieux, le processus de «naturalisation » pour nous - en prenant garde à toute dérive téléologique - peut nous préoccuper... Inversement, les concepts abstraits décrivant des processus sociaux (et non pas des résultats contingents - comme l'individu, la famille ou le marché... Les locuteurs français sont de ce point de vue « désavantagés » par rapport à un certain nombre de langues étrangères - p. ex. l'allemand - dans lesquelles processus et résultat peuvent être clairement distingués de manière suffixale. En français, les processus en question sont identifiés par les suffixes - ment ou -tion, qui néanmoins peuvent aussi signifier le résultat), et seulement eux, peuvent constituer, après définition rationnelle, les instruments de mesure et d'explication des transformations sociales historiques. En admettant qu'il faille choisir entre historicisme évolutionniste et culturalisme (cf. à propos de la notion d'« individu» Brigitte-Miriam Bedos-ReZak et Dominique Iogna-Prat, L'individu au Moyen Âge. Individuation et individualisation avant la modernité, Paris, Aubier, 2004, p. 8-9), le refus de n'importe quel historicisme évolutionniste (notamment lorsqu'il ne porte que sur des résultats contingents) ne suffit donc en aucun cas pour être ipso facto taxé de culturalisme (qui sousentend le refus d'un certain régime d'historicité et une hypostasie du passé).

7. Il s'agit du corpus de sources constitué pour l'étude de l'espace social des Thüngen dans le cadre de ma thèse (La noblesse contre le prince. L'espace social des Tbüngen à la fin du Moyen Agge (Franconie, ca. 1250-1525), Stuttgart, Thorbecke, 2000), qui rassemble quelque 8000 occurrences de termes désignant des documents écrits (cf. le détail dans «Brief und schrift. Überlegungen über die sozialen Grundlagen schriftlichen Austauschs im Spätmittelalter am Beispiel Frankens », dans : Ludolf KuCHENBUCH, Uta KLEINE (dir.), 'Textus' im Mittelalter. Komponenten und Situationen des Wortgebraucbs im schriftsemantischen Feld, Göttingen, Vandenhoeck \& Ruprecht, à paraitre).
} 
Si l'on se concentre sur ces "pages», on observe d'autre part que schrift n'est utilisé que pour un type de document, ce que nous appelons aujourd'hui 'lettres' (correspondances), tandis que ces dernières sont beaucoup plus rarement désignées comme des briefes. Si l'on examine alors le rapport entre distribution lexicale et distribution typologique, on observe que la fréquence de scbrift dans l'ensemble des termes utilisés est spectaculairement identique de la fréquence des 'lettres' dans l'ensemble du corpus étudié $(22,5 \%)^{9}$. Quant à brief, enfin, le mot désigne avant tout les documents que nous désignons aujourd'hui comme des 'chartes'. Mais comme celles-ci ne représentent qu'environ $50 \%$ de l'ensemble des documents étudiés ${ }^{10}$, cela signifie que bien d'autres types de documents que les 'chartes' utilisent le terme, très souvent pour désigner une 'charte' (p. ex. les notices de registres ou, plus épisodiquement, les annales ou chroniques), mais parfois aussi pour désigner une 'lettre'. Dans ce dernier cas toutefois, on observe que brief est massivement utilisé pour une 'lettre' lorsque celle-ci est évoquée par une 'non-lettre', c'est-à-dire aussi à la troisième personne et comme objet indéfini (ein brief), alors que schrift est plutôt utilisée par une lettre à son propre propos (meine schrift, diese schrift) ou alors à propos d'une autre lettre en rapport avec la lettre qui en parle (deine schrift, seine scbrift) ${ }^{11}$.

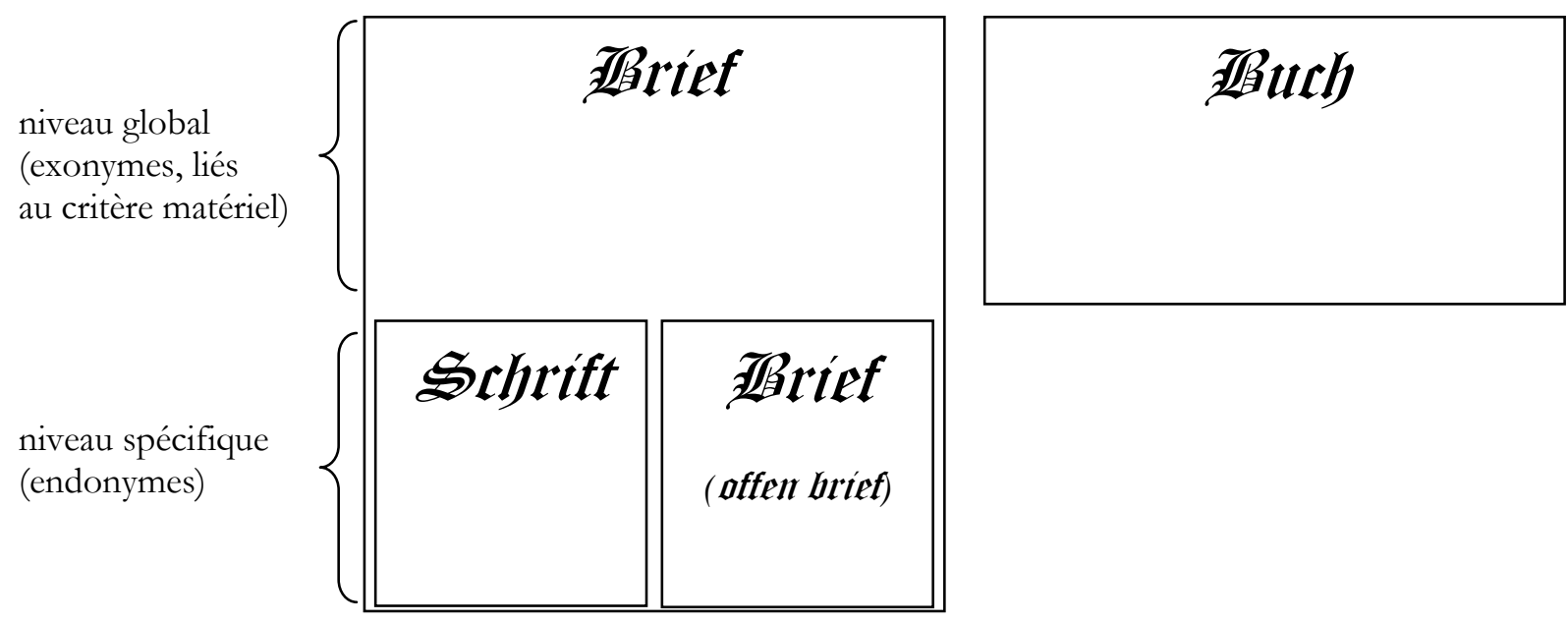

Schéma 1 : Distribution structurelle du lexique

\footnotetext{
8. Ainsi, un échange de lettres de novembre-décembre 1482 (conservé en copie au Staatsarchiv Würzburg, Thüngen-Archiv, B 9, pp.118-128), établit clairement à la fois les équivalences lexicales et les rapports de fréquence : les 11 lettres présentent 24 occurrences de schrift, par quoi les lettres s'auto-désignent ou désignent celles reçues ; 2 occurrences de scbreiben, interchangeables avec schrift (dein schreiben devient plus loin, dans les mêmes textes, dein schriefft); 6 occurrences de brief, également équivalent à schrift; 2 occurrences de klageschrift qui caractérisent le thème de la schrift que l'on envoie ("lettre de plainte/protestation»); à quoi l'on pourrait ajouter les adjectifs bescbrieben et schriftlich, accolés à des types de message (antwort, forderunge, rechtbot: « réponse », « réclamation », « sentence ») ou adverbialisés (schriflich verstehen lassen : « faire entendre par écrit »).

9. «Spectaculairement », car une telle identité est inutile à la démonstration (qui n’a besoin que d'ordres de grandeur comparables). Par ailleurs, le résultat repose sur une distribution temporelle très inégale : il n'y a aucune lettre conservée avant le début du XVe s. : le taux de $22,5 \%$ sur $1275-1525$ correspond à un taux de 25,5\% sur 1400-1525.

10. Typologie du corpus : 49,75\% sont des chartes, $22,5 \%$ des lettres, $13,5 \%$ des notices dans des registres, $9 \%$ des passages de chroniques ou annales, $3,25 \%$ des actes de procès, $1,75 \%$ des notices biographiques et $0,5 \%$ des représentations iconographiques.

11. Exemplaire de ceci est le registre d'entrées des lettres reçues du Conseil de Nuremberg de 1449-1457 (non pris en compte dans les données chiffrées fournies ci-dessus, car édité après les comptages) : Das Briefeingangsregister des Nürnberger Rates für die Jahre 1449 1457, éd. Dieter RüBSAMEN, Sigmaringen, Thorbecke, 1997. Les ‘lettres’ y sont presque toutes désignées comme des briefe, parfois avec précision du contenu (en règle générale selon le principe de composition avec brief semblable à celui rencontré n. 8 avec klageschrift, parfois simplement en tant que antwort, citacion, furderung, clag, etc. : cf. la liste de Rübsamen, p. 15) ; zettel apparait aussi, mais toutefois de manière tellement groupée (p. ex. sept lettres enregistrées les unes après les autres en 1450, p. 123), qu'on peut sans doute y voir l'effet d'une manie passagère du scribe (ou d'un changement de scribe ?). Mais si l'on prend en compte les Briefbücher (BB), registres (conservés au Staatsarchiv Nürnberg) où sont transcrites in extenso les lettres envoyées par le Conseil et dans lesquelles sont évoquées les mêmes 'lettres' reçues que dans le registre édité par Rübsamen, on observe que beaucoup de ces lettres reçues ne sont ici pas évoquées comme brief, mais comme deine ou Ibre schrift ou schreiben: cf. p. ex. RÜBSAMEN, $\mathrm{n}^{\circ} 5457$ comparé avec BB 24, f $217 \mathrm{v}$; RÜBSAMEN, $\mathrm{n}^{\circ} 5454$ avec BB 24, fo 218r ; RÜBSAMEN, $\mathrm{n}^{\circ} 6953$ avec BB 26, fo 44r; RÜBSAMEN, $\mathrm{n}^{\circ}$ 7128 avec BB 26, f ${ }^{\circ} 74 \mathrm{r}$, etc. Il ne s'agit cependant en aucun cas d'un phénomène systématique, ce qui signifie par conséquent non pas que toute lettre parlant d'une lettre (ou d'elle-même) emploie schrift/schreiben mais que toute schrift/schreiben est une autodésignation ; schrift/schreiben est donc le résultat d'un choix lexical, donc d'un acte signifiant.
} 
Le terme brief fonctionne donc à deux niveaux : l'un, global et générique, où il sert à désigner à peu près tous les types de document écrit non relié12, et l'autre, « interne », c'est-à-dire correspondant à la manière dont chaque document s'auto-désigne (niveau 'endonomastique'), où brief désigne plus spécifiquement la 'charte' (notamment sous la forme offen brief) et surtout où la 'lettre' se distingue nettement au sein de la catégorie générique brief en recourant au terme schrift - comme si, pour le dire de façon simpliste, la 'lettre' parlant d'elle-même disait: « je ne suis pas une brief comme les autres ».

L'intéressant ici est donc moins le fait que brief et schrift forment près de $100 \%$ des termes utilisés pour les écrits volants - puisque ceci est largement corrélatif de la composition du corpus -, que la différenciation menée de façon conséquente des deux termes, comme si l'on ne voulait pas mélanger les deux mots au niveau endonomastique, et donc les deux principaux types de document. Mais dès lors que le terme brief peut avoir un usage aussi global (et que la nature du document peut être, souplement, précisée par composition 'thème+brief qui, techniquement, aurait très bien pu être appliquée pour la 'lettre'), à quelle exigence renvoie le fait que les 'lettres' cherchent à se distinguer systématiquement ${ }^{13}$ du reste des briefe, construisant ainsi, au niveau interne, un écart massif entre la charte et la lettre ? Afin de pouvoir répondre à ceci, il importe également d'observer l'existence de pratiques de conservation radicalement distinctes entre brief et schrift.

\section{2) Une conservation bipolaire}

J'ai déjà évoqué la différence de fréquence typologique ('chartes' $=50 \%$, lettres $=22,5 \%$ ), dont on doit considérer qu'elle n'est pas liée aux volumes de production écrite, mais à des pratiques de conservation différentielles. Il est certainement inutile d'insister ici sur le fait que la conservation des documents écrits est tout sauf une pratique allant de soi, normale, nécessaire et rationnelle (ce qui est notre façon de voir les choses, mais des systèmes sociopolitiques hautement développés comme la Chine ancienne ont développé des systèmes très différents de conservation de la mémoire administrative - et l'on n'oubliera pas par ailleurs les cas nombreux où l'on a détruit des « originaux » après avoir confectionné des cartulaires). Si l'on ne considère pas la conservation comme quelque chose qui va de soi, c'est alors le fait d'avoir des archives qui doit nous intriguer et être expliqué ${ }^{14}$.

Or on observe là encore une nette bipolarisation. En effet, l'examen des stratégies de conservation mises en œuvre dans le cas du corpus qui a servi de base aux calculs évoqués plus haut fait apparaitre, là encore, un écart radical entre les deux types de document: d'un côté, tout laisse penser que les lettres reçues font l'objet d'une nette " négligence » (elles ne sont que lentement recopiées, et bien après les lettres envoyées ${ }^{15}$ ) et, fréquemment, d'une destruction volontaire ${ }^{16}$. De l'autre, les 'chartes' (reçues) font l'objet

12. Ne sont toutefois apparemment jamais désignés comme brief les instruments notariaux (instrument), les brouillons (note) et les cédules (zettel, annexes de 'lettres' : cf. les Briefbücher nurembergeois).

13. Cette «systématicité» ne doit pas être comprise au sens absolu (au sens de «toujours») mais au sens propre (au sens de «de manière systémique ») : j’ai déjà signalé l'existence de cas où la 'lettre' est désignée comme brief (y compris de manière endonomastique) et j'ai parlé plus haut de "différenciation menée de façon conséquente ». D’une manière générale, il me semble toujours plus intéressant qu'existent des contre-exemples car ceux-ci sont le signe que ce à quoi ils correspondent était possible dans la société considérée mais est resté marginal. C'est là le fameux principe de "l'exception qui confirme la règle », dont Maurice Godelier, L'idéel le matériel. Pensée, économies, sociétés, Paris, Fayard, 1984, p. 224-225, donne une lecture anthropologique.

14. Ces problèmes de conservation ont été, entre autres, abordés dans le cadre de la journée de l'École doctorale d'histoire de Paris consacrée à "L'historien et 'ses' 'sources'" (cf. Hypothèses 2003. Travaux de l'École doctorale d'bistoire de l'Université Paris I PanthéonSorbonne, Paris, Publications de la Sorbonne, 2004, p. 271-362) et dans l'ensemble des articles consacrés au thème "Fabrique des archives, fabrique de l'histoire » (Revue de Synthèse, 125 (2004), p. 1-195).

15. Cet écart temporel peut bien sûr s'expliquer par le fait que, dans la mesure où les lettres envoyées ne sont plus disponibles (puisqu'elles sont envoyées), leur enregistrement est un moyen d'en conserver la trace alors qu'il est inutile de recopier des lettres reçues, pour lesquelles seuls des registres sommaires, permettant de retrouver la lettre cherchée, sont utiles - en même temps qu'ils témoignent de ce qu'on conserve les lettres. À Nuremberg, le plus ancien registre d'entrées de lettres, sous la forme de notices très résumées, date du milieu du $\mathrm{XV}^{\mathrm{e}} \mathrm{s}$. alors que les lettres envoyées sont enregistrées depuis le début du siècle, et ce registre reste isolé jusqu'à 1490 : RÜBSAMEN, Briefeingangsregister..., p. 12. L'absence d'autres registres avant 1490 est en général expliqué par leur destruction accidentelle (RÜBSAMEN, ibid.), qui est cependant étonnante en contexte nurembergeois et ne me convaint guère. On pourrait bien sûr considérer que la production et la conservation du registre aient été purement conjoncturelles, puisqu'il débute en 1449 avec les tensions croissantes qui débouchent sur la Guerre margraviale de 1449-1452. Toutefois, l'enregistrement ne s'arrête pas avec la guerre et est poursuivi jusqu'en 1457 et presque jusqu'à la fin du registre (RÜBSAMEN, $p$. 18) : ce registre semble donc bien se replacer dans une série dont lui seul est conservé. Mais comme tout semble montrer que ce registre n'était pas le premier de la série (cf. Laurence BuCHHOLZER, L'intercommunalité en Franconie à la fin du Moyen Áge, Lyon II, thèse de doctorat inédite, 2001, p.143, qui mentionne la réalisation en 1439 d'« un registre de toutes les Briefe, Schriften et Bücher [NB : remarquer la typologie de base employée, à rapprocher du schéma 1 !] qui sont conservées dans la salle et son annexe voûtée »), donc que tant les registres avant qu'après 1449-1457 ont disparu, j'aurais plutôt tendance à penser que l'accident est moins la destruction des registres que la conservation, exceptionnelle, du registre de 1449-1457, probablement en raison des informations 
d'un soin et d'une préservation attentifs, en original, en copie et à l'aide de listes d'inventaire ${ }^{17}$. Une telle conservation spécifique a nécessairement entrainé une sous-représentation lexicale de schrift-mais surtout, elle vient renforcer la bipolarité du champ des pratiques documentaires. Mais pourquoi donc la 'charte' est-elle tendanciellement conservée tandis que la 'lettre' est tendanciellement non conservée ? Et si l'on considère que conserver est le résultat d'un choix, pourquoi alors la 'charte' est-elle ainsi distinguée de la 'lettre'? Bref, du point de vue terminologique comme de la conservation, à quoi renvoie l'écart spécifiquement instauré entre 'charte' et 'lettre', offen brief et schrift?

\section{3) L'élimination des critères rétrojetés du public/privé et de l'utilité}

On pourrait d'emblée considérer que le binôme charte/lettre recouvre le binôme public/privé18. De fait, on sait bien que la charte prétend s'adresser à tout le monde (" à tous ceux qui ces lettres verront, etc. »), tandis que l'adresse de la lettre est plus définie. Mais outre les problèmes que pose en principe le recours au binôme moderne public/privé19, on voit bien que son application au problème qui nous occupe est inadéquate : lorsque le Conseil de Nuremberg écrit une lettre, p. ex., au Conseil d'Augsbourg, ou au margrave de Brandebourg, s'agit-il vraiment d'un acte privé ? C'est d'ailleurs la raison pour laquelle les historiens ont été amenés à forger au XIXe s. la notion de Privatbrief, « lettre privée », pour désigner spécifiquement les correspondances circulant au sein de la famille ou entre amis ${ }^{20}$, c'est-à-dire relevant de la sphère du privé selon les critères contemporains. Par ailleurs, les diplomatistes classent un certain nombre de 'chartes' dans la catégorie des Privaturkunden, dès lors qu'elles ont été émises par une personne privée, ne représentant qu'elle-même (émettant donc un " acte sous seing privé », comme on dit aujourd'hui, comme un acte de vente, une reconnaissance de dette, un serment de non-agression, etc.) ${ }^{21}$. Pourtant, les 'lettres' du Conseil sont bien des schriften, tandis que les 'chartes' de particuliers se désignent comme briefe (y compris offen briefe, y compris créditées de la kraft und macht/virtus de toutes les briefe)... Notre distinction schrift/ brief n'a donc très probablement rien à voir avec la distinction privé/public.

On pourrait alors, arguant de ce que seul serait conservé ce qui pouvait être utile tandis que serait jeté ce qui était devenu inutile dès lors qu'il avait été lu, considérer que c'est le critère d'utilité qui intervenait. Mais il ne s'agit là certainement que d'une fausse évidence. D'une part, cela revient à mobiliser nos critères de l'utilité et à les rétrojeter sur une autre société, comme s'il existait des constantes du comportement humain. Or il est plus que probable que régnait, dans la société médiévale, une autre conception de

concernant la période 1449-1450 qu’il recelait (on retrouverait là le rapport entre fixation écrite de la mémoire et les périodes de « crise » déjà souligné par Gerd Althoff, «Zur Verschriftlichung von Memoria in Krisenzeiten », dans : Dieter GeUENICH, Otto Gerhard OexLe (dir.), Memoria in der Gesellschaft des Mittelalters, Göttingen, Vandenhoeck \& Ruprecht (Veröff. d. Max-PlanckInstituts f. Geschichte, 111), 1994, p. 56-73). Et si l'on considère que ces registres d'entrées n'avaient d'utilité qu'avec les lettres qu'ils enregistraient, donc que la conservation des registres suppose la conservation des lettres, leur destruction pourrait laisser entendre que les lettres ont été alors détruites - mais même l'édition de RÜBSAMEN, Briefeingangsregister..., semble montrer, pour le registre d'entrées conservé, que les lettres reçues par le Conseil n'ont pas été préservées (sans que la date de destruction puisse cependant être assurée), puisque Rübsamen n'est pas en mesure de mettre en rapport les mentions du registre avec les lettres correspondantes, mais seulement avec leur mention dans les lettres envoyées par le Conseil et enregistrées dans les Briefbücher déjà mentionnés. Le cas spécifique des lettres envoyées par les Thüngen a été présenté dans mon ouvrage La noblesse contre le prince..., p. 27, et Christoph von BRANDENSTEIN, Urkundenwesen und Kanzlei, Rat und Regierungssystem des Pfälzer Kurfürsten Ludwig III. (14101436), Göttingen, Vandenhoeck \& Ruprecht (Veröff. d. Max-Planck-Instituts f. Geschichte, 71), 1983, p. 21, constatait aussi (mais sans l'expliquer), à propos des lettres envoyées par les comtes palatins du Rhin, qu'elles sont beaucoup moins bien conservées que leurs chartes - dans tous les cas, tout ceci me semble suggérer une relative négligence vis-à-vis des lettres.

${ }^{16}$ Cf. l'exemple de 1512 à propos de Friedrich VIII von Thüngen mentionné dans mon ouvrage La noblesse contre le prince..., p. 27 (repris dans mon article «Ce qu'écrire veut dire au Moyen Âge... Observations préliminaires à une étude de la scripturalité médiévale ", Memini. Travaux et documents de la Société d'études médiévales du Québec, 4 (2000), p. 5).

17. Sur les cartulaires, les listes de chartes et l'archivage des chartes, cf. Olivier GuYOTJEAnnin, Laurent MorelLe, Michel ParisSE (dir.), Les cartulaires. Actes de la table ronde de Paris (5-7 décembre 1991), Paris/Genève, Droz/Champion (Mémoires et documents de l'École des Chartes, 39), 1993 ; Olivier Guyotjeannin, Jacques Pycke, Benoît-Michel Tock, Diplomatique médiévale, Turnhout, Breplos (L’atelier du médiéviste, 2), 1993, p. 296-307 ; « Fabrique des archives, fabrique de l’histoire », op. cit., ; MORSEL, La noblesse contre le prince..., p. 27-28.

18. Jérôme HAYEZ, «Avviso, informarione, novela, nuova : la notion d'information dans les correspondances marchandes toscanes vers 1400 », dans : Claire Boudreau, Kouky Fianu, Claude GAuvard, Michel HÉBerT (dir.), Information et société en Occident à la fin du Moyen Age. Actes du colloque international tenu à l'Université du Québec à Montréal et à l'Université d'Ottawa (9-11 mai 2002), Paris, Publications de la Sorbonne, 2004, p. 131-132, considère ainsi la lettre marchande médiévale comme une forme d'appropriation privée/personnelle de données publiques.

19. En vertu des mêmes considérations que celles évoquées n. 7 .

20. Cf. Georg STEINHAuSEN, Deutsche Privatbriefe des Mittelalters, 2 vol., Berlin, 1899-1907.

21. Cf. Friedrich BECK, Eckhart HeNNING (dir.), Die archivalischen Quellen : eine Einführung in ihre Benützung, 2e éd., Weimar, Böhlau, 1994, p. 28 ; Gerhard Theuerkauf, Einfübrung in die Interpretation historischer Quellen - Schwerpunkt: Mittelalter, Paderborn/München/Wien/Zürich, Schöningh, 1991, p. 117. 
l'utilité - et de toute façon, il vaut mieux faire comme si c'était le cas plutôt que l'inverse. En effet, d'autres critères de conservation pourraient être intervenus, comme la personnalité de l'auteur, la beauté du style, l'intérêt particulier du contenu, etc., c'est-à-dire des motifs liés au contexte de l'émission. Par ailleurs, on voit bien que dans une société utilitariste comme la nôtre, la lettre jouit pourtant d'une valeur sociale reconnue, comme le montrent les nombreuses publications de correspondances - ce qui implique la conservation des lettres par les destinataires puis leurs héritiers. Qui plus est, notre société repose sur un principe de conservation absolue, non pas en vertu du principe d'utilité mais par vénération de l'actuel qui deviendra vieux : nous ne conservons pas parce que c'est utile, mais parce que c'est ou ce sera historique. Mais quelle est l'utilité de l'histoire ? Pourquoi, alors, considérer que l'utilité soit nécessairement un critère pertinent pour la société médiévale ? D’une manière générale, on peut considérer que l'on conserve ce qui a de la valeur - mais la formation de la valeur est un processus profondément ancré dans la logique sociale de chaque société, que la rétrojection de nos critères de valeur ne peut que rendre inintelligible.

Le recours à des schémas explicatifs extérieurs (qui plus est probablement anachroniques) ne parait donc guère pertinent et il est certainement plus efficace de considérer que les modalités lexicales et de conservation distinctes de la brief et de la schrift renvoient non à leur finalité de notre point de vue mais au statut social spécifique de ces deux types de document - c'est-à-dire que les pratiques terminologiques et de conservation sont la manifestation en même temps que la réalisation d'une structure (socio)logique. Ceci sous-entend que le nom et le mode de conservation étaient le résultat de la procédure de différenciation, non pas en tant qu'effets subsidiaires, que conséquences externes - liées notamment à l'intentionnalité des acteurs, qui décident de nommer ainsi ou de garder ou non les documents -, mais en tant que réalisations de propriétés internes des documents - produits en tant que brief et ipso facto à conser$\operatorname{ver}^{22}$ ou en tant que schrift et ipso facto non conservable.

\section{4) Structures de la communication médiévale (en Franconie à la fin du Moyen Age)}

Il s'agit donc de comprendre la différence typologique entre brief et schrift non pour comprendre ce qu'est d'une part, une brief et, d'autre part, une schrift, mais quel est le sens de la distinction menée conséquemment entre les deux types, le sens d'une différence non pas factuelle mais visiblement construite. Ceci implique néanmoins de comprendre en quoi ces deux types de documents se différencient absolument, c'est-à-dire précisément ce que réalisait la distinction terminologique et de conservation. C'est là que nous retrouvons l'objet de notre réunion, les rapports de communication, que ces deux types de document configurent de manière radicalement distincte comme le montre leur examen structural.

Dans le cas de la 'charte', d'un côté, un émetteur [1] donne à lire/voir/entendre à un public indéfini et illimité [3] un document qui concerne une personne (physique ou morale) qui en est le bénéficiaire [2] ; l'émetteur [1] scelle le document et le donne au bénéficiaire [2], qui en devient le détenteur et se chargera concrètement, le cas échéant, de faire lire le document au public [3]. La 'charte' est donc fondamentalement un document qui instaure par principe un rapport de communication tripolaire, fondé sur la dissociation du récepteur du document [2] et du récepteur du message [3] en même temps que sur la dissociation des deux donneurs-à-lire, l'émetteur [1] et le détenteur [2]. Par ailleurs, on observe qu'aucune réponse n'est demandée, l'information ne circule que dans un seul sens. On n'est donc pas dans une structure dialogique - ce qu'expriment bien les personnes du verbe employées : première personne [1] vs. troisièmes personnes [2] et $[3]^{23}$.

\footnotetext{
22. Des travaux à ce propos sont d'autant plus nécessaires que les chartes constituent encore aujourd'hui à la fois une grosse masse des documents médiévaux conservés et, conséquemment, l'un des principaux soubassements archivistiques sur lesquels repose le travail des historiens. Il serait absolument nécessaire d'évaluer dans quelle mesure la compréhension que les historiens ont de la société médiévale est déterminée non pas par le caractère aléatoire de la conservation des archives, mais par les effets documentaires de choix médiévaux, nécessairement sociaux et qui orientent (voire biaisent) donc le travail historique. Le cas du Trésor des chartes français a été bien étudié et permet de percevoir les effets spécifiquement historiographiques liés à un fonds de cet ordre : cf. Olivier GuYotjeAnnin, Yann Potin, «La fabrique de la perpétuité. Le Trésor des chartes et les archives du royaume (XIII $\mathrm{XIX}^{\mathrm{e}}$ s.) », Revue de Synthèse, 125 (2004), p. 15-44. Mais j'irai même plus loin, car dans le cas du Trésor des chartes, la destruction des archives de la cour des comptes a fait disparaitre le caractère « conservé » du Trésor des chartes, pour en faire « ce qui reste »; mais là encore, le fait même qu'il y ait eu un "reste » (le Trésor des chartes) est significatif, comme devrait l'être considérée l'existence de chartriers dans les dépôts d'archives.

${ }^{23}$ Le caractère ternaire de ce schéma pourrait apparaitre bien simpliste au regard des divers acteurs que distingue la diplomatique (auteur, disposant, destinataire, bénéficiaire, impétrant, rédacteur, scribe selon Olivier GUYOTJEANNIN, Jacques PYCKE, BenoitMichel Tock, Diplomatique médiévale, Turnhout, Brepols (L'atelier du médiéviste, 2), p. 25, à laquelle Béatrice FrAENKEL ajoute encore les témoins («L'auteur et ses signes», dans : Michel ZimmermanN (dir.), Auctor et auctoritas. Invention et conformisme dans l'écriture médiévale. Actes du colloque de Saint-Quentin-en-Yvelines (14-16 juin 1999), Paris, École des Chartes, 2001, p. 417). Mais d'une part, comme le signalent les auteurs mentionnés, il s'agit là de la situation analytique la plus étendue, dans la mesure où soit cer-
} 

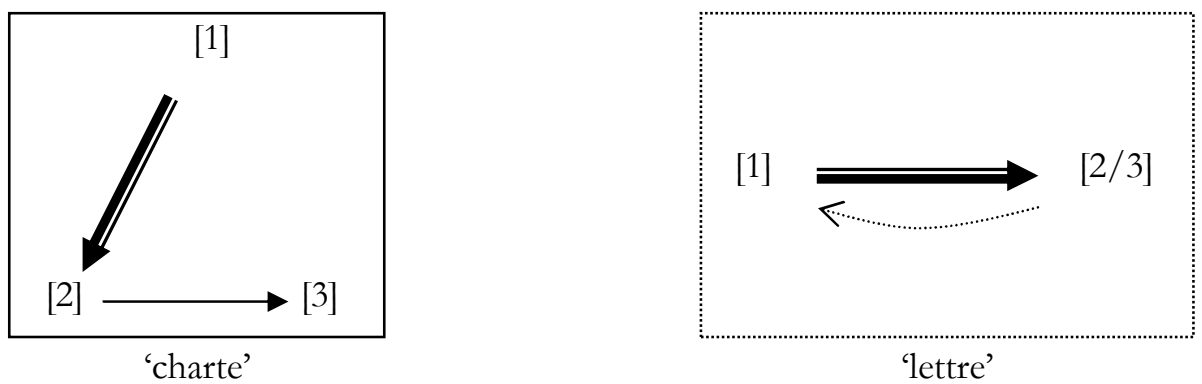

'lettre’

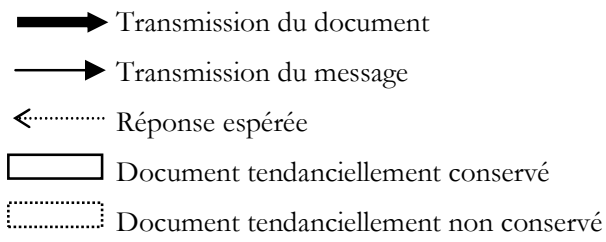

\section{Schéma 2 : Structures de communication de la 'charte' et de la 'lettre'}

Face à cela, la 'lettre' établit une structure bipolaire : le destinataire de la lettre et celui du message sont identiques en face de l'émetteur $([3=2] v$ s. [1]). En outre, la schrift se situe d'emblée dans un échange circulaire, puisque dans la quasi-totalité des cas, l'émetteur termine en signalant qu'il attend la réponse, laquelle commence le plus souvent par la formule du genre «J'ai/nous avons bien reçu ta/votre lettre... » et résume la plupart du temps la teneur de la lettre à laquelle elle répond ${ }^{24}$. La circularité de l'échange est ainsi

tains de ces acteurs n’interviennent que de manière épisodique, soit peuvent être généralement identiques (p. ex. bénéficiaire et destinataire), soit disparaissent; d'autre part, cette nomenclature concerne spécifiquement l'émission (rapport [1]/[2] dans notre schéma) mais pas la structure de communication en tant que telle, comme le montre l'absence de toute prise en compte du public, pourtant mentionné le plus souvent dans l'adresse; enfin, comme nous y invite d'ailleurs l'usage à ce propos de la notion d'« actants » par B. Fraenkel (op. cit., p. 415-417), on pourra se rappeler que le schéma actantiel d'Algirdas J. Greimas réduit luimême le grand nombre de positions spécifiques («fonctions» morphologiques, au nombre de 31, dont certaines facultatives) identifiées par Propp à trois paires d'actants : un triolet correspond à l'axe de la communication (destinateur-sujet-destinataire) et le second à l'axe du pouvoir (adjuvant-objet-opposant), tandis que l'articulation des deux axes est assurée par l'axe du désir reliant le sujet et l'objet. Il ne saurait être question d'appliquer mécaniquement le schéma actantiel à notre propos (bien que l'axe de communication présente un ensemble de phases du plus haut intérêt du point de vue analytique) mais simplement de souligner que la multiplication des acteurs imposée par la nécessité de rendre compte de la complexité institutionnelle ne doit pas faire disparaître la logique interne au processus de communication - complexité institutionnelle dont on pourrait même considérer qu'une partie de son sens résidait précisément dans la nécessité sociale (propre à toutes les sociétés) de voiler les rouages de la domination sociale (ce qui reviendrait alors à prendre le contre-pied de l'interprétation de B. Fraenkel, op. cit., p. 418, qui corrèle la multiplicité des actants à «la difficulté [médiévale] à construire les conditions de félicité nécessaires à la performativité des actes écrits en général », confondant ainsi, comme il en va très souvent dès lors que l'on parle du Moyen Âge, le savoir-faire et le vouloir-dire). Mais cf. infra, n. 35, pour une observation importante qui pourrait conduire à une adaptation du présent schéma ternaire.

${ }^{24}$ Cf. p. ex. les lettres envoyées par le Conseil de Nuremberg à Balthasar von Thüngen les 20 août et 3 septembre 1439 : Bathazarn von Tüngen. Lieber der von Tüngen, als ir uns auf die scbrifft, so wir den von Sültzvelt nehst getan baben von wegen Jacob Hewglins, unsers burgers und etlichen weingerten zu Sützvelt, verscbriben habt in lengern worten, wie der zu Sültzvelt, der sölliche güter ynnenhab, geantwortt hab, er hab sölliche güter gekawfft und er meyne, daz in Hewglin daruber pillich on red ließ, doch so seyet ir sein mecbtig ₹u unvertzogem rechten zu Sültzvelt etc., das baben wir wol vernomen. Also... [à la fin :] ... und lat uns daruber ewer verschriben antwort wider wissen bey disen botten (Staatsarchiv Nürnberg, BB 14, $\left.\mathrm{f}^{\circ} 27 \mathrm{r}\right)$. Balthazar von Tungen. Lieber der von Tüngen, als ir uns auf unser nebst schrifft von Jakoben Hewglins und Hanns Soldners, unser burgere, wegen verschriben und geantwortt habt, wie ir den von Sültvvelt söllicher sach halben gescbriben und den genanten unsern nehsten burger mitgesandt und die dem vorgenanten Soldner beid geantwortt habet, der denn ewer erberkeit geredet, die dartzu tragen etc., das baben wir wol vernomen. Und... (ibid., $\mathrm{f}^{\circ} 34 \mathrm{r}$ ). À l'inverse (et logiquement), on trouve des lettres de déclaration de guerre (qui ont la forme d'une lettre), qui déclarent expressément qu'aucune réponse n'est attendue (p. ex. Staatsarchiv Würzburg, Thüngen-Archiv, B 9, p. 157) ; c'est probablement ce qui explique que certaines de ces lettres de faide prévoyaient explicitement la restitution de la lettre à son émetteur une fois la réconciliation établie (cf. pour le cas autrichien Otto BRUNNER, Land und Herrschaft. Grundfragen der territorialen Verfassungsgeschichte Österreichs im Mittelalter, 4e éd. Wien/Wiesbaden, Rudolf Roher, 1959, p. 74). 
bien visible et correspond à une structure dialogique : les pronoms personnels employés sont également différents (je/nous vs. tu/vous) et,. Les deux types de document paraissent donc opposés strictement en tout ${ }^{25}$.

La distinction et bipolarisation menées de façon conséquente au niveau terminologique renvoient donc à une distinction et bipolarisation nettes au niveau des rapports structuraux de communication - à quoi l'on pourrait ajouter l'instauration par la 'charte' d'une communication dans le temps (dans son second segment [2]-[3]) alors que la schrift se conçoit comme une communication dans l'espace et selon toute vraisemblance synchronique (comme palliatif à un dialogue en coprésence, au-delà des simples problèmes techniques d'acheminement de la lettre ${ }^{26}$. Reste cependant à comprendre de quelle manière la conservation spécifique de la 'charte' s'articule avec la structure de communication qu'elle réalise. La formulation de cet impératif repose, je le rappelle, sur trois hypothèses : 1) c'est la conservation qui est signifiante (et non pas un fait en soi, une pratique commune et naturelle) ; 2) cette conservation n'est pas liée au critère de l'utilité (qui n'est qu'un faux-semblant) mais 3) constitue une propriété de la 'charte', d'emblée produite comme un « document-à-garder » (au lieu de considérer la conservation comme un «accident » du document, comme quelque chose qui arrive ou n'arrive pas à tel ou tel document).

\section{III.- Hypothèse d'interprétation : l'enjeu de la réponse}

La double dissociation (émetteur du document/dépositaire du document, récepteur du document/récepteur du message) place deux « donnant-à-lire » perpétuels (l'émetteur [1], qui donne à lire une fois pour toutes, définitive, et le destinataire/dépositaire du document [2], susceptible de produire et faire lire la lettre, à volonté dans le futur) en face du récepteur du message [3], placé dans une situation de pure réception. C'est cette structure qui fait la 'charte' (par opposition à la 'lettre', et non par conformité à un impératif juridique comme l'impossibilité de réaliser autrement un acte écrit prétendument 'public' - puisque d'autres formes d'actes sont apparues pour matérialiser les actions dont traitent les chartes), et c'est donc à ce rapport entre faire-lire et lire-seulement qu'il faut s'intéresser.

\section{1) La centralité de la réponse dans la définition de l'Öffentlichkeit}

C'est là que nous retrouvons notre problème de départ, celui de l'Öffentlichkeit. La 'charte', bien qu'elle prétende instaurer un rapport de communication entre un émetteur singulier et un public (la 'charte' est le plus souvent dite offen et déclare souvent les choses offenlich) illimité (allermeniglich), semble tout à fait correspondre à la conception que se faisait et relayait Habermas de l'espace social médiéval, à savoir un espace social saturé d'autoreprésentations du pouvoir. Toutefois, ce que la distinction fondamentale entre 'charte' et 'lettre' me semble faire apparaître, c'est que le problème réside moins dans l'idée de la représentation plus ou moins ritualisée des arcanes du pouvoir (la messe en latin) que dans l'impossibilité de la réponse.

25. La discussion lors de la journée d'études du 31 mai a fait apparaittre le risque de malentendus autour de la notion de « réponse » : me plaçant dans une perspective structurale, je ne prends pas en compte le fait que l'émission d'une 'charte' puisse constituer une « réponse » implicite (et métaphorique) à une difficulté ou une nécessité sociale propre au milieu dans lequel cette émission se produit. Ce qui m'intéresse ici, c'est que la charte est structurée comme une émission close sur elle-même : l'exposé des motifs de l'émission explicite certes les conditions de l'émission, mais ne fait en aucun cas de la 'charte' une réponse au sens propre du terme, et elle n'appelle clairement aucune réponse.

26. Il faudrait donc corriger l'analyse établie dans «Brief und Scbrift... », où j'écrivais «1) Der brief ist zum Übertragen bestimmt und wird (tendenziell) aufbewahrt ; die Kommunikation erfolgt räumlich und zeitlich. 2) Die schrift ist zum Übertragen bestimmt und wird (tendenziell) nicht aufbewahrt; die Kommunikation erfolgt räumlich. 3) Das register ist nicht zum Übertragen bestimmt und wird (tendenziell) aufbewahrt ; die Kommunikation erfolgt zeitlich » : la différence entre 'charte' et 'lettre' serait plutôt que, d'un côté, la 'charte' est nécessairement transmise dans l'espace (de l'émetteur au bénéficiaire, d'où la mention du lieu d'émission en même temps que la date, puisque le lieu de conservation, par le bénéficiaire, à partir duquel la charte sera utilisée, est imprévisible, de même que le moment auquel elle le sera) mais la communication qu'elle instaure se déroule dans le temps; de l'autre côté, la 'lettre' instaure une communication synchronique dans l'espace (la 'lettre' est conçue comme une fiction de communication de face-à-face, que seul l'éloignement rend impossible : cf. John VAN ENGEN, «Letters, Schools, and Written Culture in the Eleventh and Twelfth Centuries ", dans : Johannes FRIED (dir.), Dialektik und Rhetorik im früberen und bohen Mittelalter. Rezeption, Überlieferung und gesellschaftliche Wirkung antiker Gelebrsamkeit vornebmlich im 9. und 12. Jabrbundert, Munich, Oldenbourg, 1997, p. 107-109 et 113114 ; de nos jours, la lettre peut fonctionner comme forme d'abolition de la distance entre l'émetteur et le destinataire : Marlène Albert-Llorca, «Le courrier du Ciel », dans : Daniel FABRE (dir.), Écritures ordinaires, Paris, P.O.L., 1993, p. 208) dont la transmission se déroule nécessairement dans le temps (durée aléatoire des transmissions, d'où la mention de la date puisqu'il s'agit d'une donnée imprévisible). Schématiquement, on aurait donc : communication à but temporel×transmission pratique dans l'espace ('charte') vs. communication à but spatialXtransmission pratique dans le temps ('lettre'). 
Je ne veux pas dire que dans la société médiévale, c'est la 'lettre' qui instaure l'espace public, mais plus abstraitement que c'est dans la différence entre la 'lettre' et la 'charte' que réside la potentialité d'un espace public. Parce que la société médiévale est «capable» de construire, au plus tard à partir du XI siècle, un système documentaire fondé sur la bipolarité 'charte'/'lettre', correspondant à (c'est-à-dire réalisée par) des structures de communication, des usages terminologiques et des modes de conservation distincts, elle recèle la possibilité d'émergence d'un espace public, fondé sur la possibilité de réponse.

Ce n'est donc pas, contrairement à Habermas, dans la prise de parole publique (mais en-dehors de la Staatssphäre) et articulée et dans l'exercice collectif du raisonnement (öffentliches Räsonnement ${ }^{27}$ que je vois l'espace public, mais dans la possibilité de réponse. Moins dans le droit d'expression (opposé à la censure $^{28}$ ) que dans le droit de réponse (opposé au dogme). Et ce qui caractérise les lieux d'élaboration de l'espace public moderne de Habermas (cafés, clubs, salon, etc.), c'est avant tout qu'il s'agit de lieux de discussion et de controverse où la réponse est possible - et c'est cette possibilité de réponse qui en fait des lieux de discussion et de controverse. Et à l'inverse, ce qui ruine l'espace public actuel, c'est l'impossibilité de réponse, liée au type de média qui devient le support du débat politique, la télévision notamment...

On retrouve ici une hypothèse-clé d'un autre philosophe, exactement contemporain de Habermas et qui inscrit aussi son analyse du sens politique de la communication dans un contexte intellectuel marxiste, avec néanmoins une orientation sémiologique et anthropologique très marquée, caractéristique du paysage scientifique français de la fin des années 1960 : Jean Baudrillard. Dans son livre intitulé Pour une critique de l'économie politique du signe, paru en 1972 et reposant sur des travaux qu'il avait publiés en 19691970 et dans lesquels on ne trouve sauf erreur nul écho des travaux de Habermas, Baudrillard fait de la réponse l'enjeu-clé de la communication au sens propre du terme, c'est-à-dire de l'échange ${ }^{29}$ :

«Toute l'architecture actuelle des media se fonde sur cette dernière définition : ils sont ce qui interdit à jamais la réponse, ce qui rend impossible tout procès d'échange (sinon sous des formes de simulation de réponse, ellesmêmes intégrées au procès d'émission, ce qui ne change rien à l'unilatéralité de la communication). C'est là leur véritable abstraction. C'est dans cette abstraction que se fonde le système de contrôle social et de pouvoir.

Pour bien comprendre ce terme de réponse, il faut l'entendre dans un sens fort, et pour cela se référer à ce qui en est l'équivalent dans les sociétés « primitives »: le pouvoir est à celui qui peut donner et à qui il ne peut pas être rendu. Donner, et faire en sorte qu'on ne puisse pas vous rendre, c'est briser l'échange à son profit et instituer un monopole : le procès social est ainsi déséquilibré. Rendre, au contraire, c'est briser cette relation de pouvoir et instituer (ou restituer), sur la base d'une réciprocité antagoniste, le circuit de l'échange symbolique. Il en est de même dans la sphère des media : il y est parlé, et fait en sorte qu'il n'y puisse nulle part être répondu.»

On le voit, Baudrillard se focalise sur la rupture entre l'échange direct et le média de masse (comme d'ailleurs Habermas ${ }^{30}$ ) alors que dans notre cas, celui de la 'lettre' et de la 'charte', il s'agit toujours d'une communication écrite : ce n'est donc pas la technologie employée qui fonde la capacité ou non de réponse. Le problème de la masse n'est pas à exclure au motif que la technologie des medias de masse n'existait pas (la 'charte' prétend toujours s'adresser à tout le monde), de même que n'est sans doute pas pertinent le problème de la distance technologiquement instituée entre l'émetteur et le récepteur (qui viendrait corroborer le fait que l'Öffentlichkeit habermassienne est née dans les salons, clubs et café, c'est-à-dire des lieux de coprésence, de démocratie langagière directe) : la distance est consubstantielle à la 'lettre' et non pas à la 'charte', tandis que les spectacles d'autoreprésentation du pouvoir se font en direct... L'hypothèse de

\footnotetext{
27. Et donc dans la disparition de ce raisonnement critique, au profit du «prêt-à-penser » qui fait de l'opinion publique une simple opinion commune, la disparition de l'espace public proprement dit.

28. L'examen par Habermas du cas français (Strukturwandel, p. 79) montre bien qu'il conçoit l'Öffentlichkeit comme contradictoire avec la censure.

29. Pour une critique de l'économie politique du signe, Paris, Gallimard, 1972, p. 208-209.

30. Habermas envisage lui aussi le problème de la non-réponse mais, de façon significative, il ne s'y attarde guère : «Funk, Film und Fernsehen bringen den Abstand, den der Leser zum gedruckten Buchstaben einhalten muß, gradweise zum Verschwinden eine Distanz, die die Privatheit der Aneignung ebenso verlangte, wie sie die Öffentlichkeit eines räsonierenden Austausches über das Gelesene erst ermöglichte. Mit den neuen Medien ändert sich die Kommunikationsform als solche ; sie wirken darum, in des Wortes strikter Bedeutung, penetranter als die Presse je es vermochte. Das Verhalten des Publikums nimmt unter dem Zwang des 'Don't talk back' eine andere Gestalt an. Die Sendungen, die die neuen Medien ausstrahlen, beschneiden, im Vergleich zu gedruckten Mitteilungen, eigentümlich die Reaktion des Empfängers. Sie ziehen das Publikum als Hörende und Sehende in ihren Bann, nehmen ihm aber zugleich die Distanz der 'Mündigkeit', die Chance nämlich, sprechen und widersprechen zu können. » (Strukturwandel, p. 187-188).
} 
Baudrillard n'est donc pas pertinente au niveau de l'opposition entre communication de masse et communication directe, c'est-à-dire entre média et absence de média (immédiateté).

En revanche, elle met en valeur le problème de la réponse, à laquelle il faut nous intéresser de plus près puisque nous avons vu que la distinction entre les deux types de document repose sur la possibilité ou non d'une réponse. Chez Baudrillard, le schéma de base repose sur l'idée de la communication conçue comme un échange (avec une nette coloration substantialiste, comme si le message était une chose) et l'application à cet échange de la théorie du don/contre-don ${ }^{31}$. On sait que cette théorie du don/contredon est difficilement transférable sur la société médiévale en raison du principe dominant de caritas, qui impose de donner gratuitement, sans attendre en retour ${ }^{32}$. Surtout, rien n'indique dans le cas de la 'charte' que le modèle du don soit pertinent pour expliquer l'émission : certes, la charte est dite "donnée » (geben, lat. datum), mais cette remise ne concerne que le rapport entre l'émetteur [1] et le bénéficiaire [2], tandis que le bénéficiaire/dépositaire n'apparait jamais (et son intérêt ne l'y pousse jamais) comme l'émetteur du message, dont il ne se présente que comme le relais.

Si donc l'impossibilité de répondre apparaît bien comme le facteur-clé de la domination idéologique, c'est - du moins pour le Moyen Âge que j'étudie - moins par le biais de la technologie médiatique qu'elle agit et moins sur la base d'un échange inégal. L'une des pistes à suivre pour comprendre la signification sociale de la non-réponse (c'est-à-dire ce qui fait de la non-réponse la mise en œuvre d'un rapport de domination) est sans doute de tenir compte des autres structures formelles de la 'charte' (ternarité, jeu des personnes), en considérant que ce sont elles qui interdisent la réponse. La non-réponse n'est en effet pas décrétée par la 'charte' et ne se déduit que de la confrontation avec la 'lettre' - ce qui signifie qu'elle est produite au sein de la 'charte'.

Du point de vue des personnes grammaticales, on doit sans doute tenir compte du fait que la troisième personne est, en linguistique structurale, le marqueur et l'institution d'une absence ${ }^{33}$. On peut ainsi considérer que la 'charte' "met en absence », dans le cadre de la communication, les destinataires tant du document que du message, tandis que l'émetteur est doublement présent, à la fois par le «je » et par le sceau, qui en est une véritable forma substantia ${ }^{34}$. Mais la structure ternaire module le sens de la mise en absence : celle du bénéficiaire [2] reçoit une signification différente de celle des destinataires du message [3], puisqu'elle lui permet de n'apparaître que comme un relais neutre, un pur représentant - en tant que celui à travers lequel l'émetteur [1] redevient présent.

\section{2) Une impossibilité idéologique de la réponse à la charte?}

La non-réponse consubstantielle à la charte fonde ainsi à la fois 1) le pouvoir de l'émetteur face aux destinataires, symboliquement privés de toute existence autonome et transformés en objets, et 2) le pouvoir du dépositaire face aux destinataires du message, face auxquels le dépositaire est dans une situation de pure

\footnotetext{
31. Chez Habermas, on observe aussi une tendance au substantialisme, dans la mesure où la circulation des idées est conçue de façon parallèle à la circulation des biens marchands. Là est probablement la différence majeure entre Habermas et Baudrillard : le premier applique à la communication équilibrée le modèle de l'échange marchand (dans le cadre de ce qu'il appelle l'Öffentlichkeit bourgeoise ; l'échange équilibré disparait lorsque l'échange lui-même devient un bien marchand : Strukturwandel, p. 180-181), tandis que le second lui applique le modèle du don/contre-don - sans exclure que Baudrillard se conçoive comme un prolongement de Habermas : l'Öffentlichkeit bourgeoise étant morte, seul le modèle du don pourrait instaurer une nouvelle Öffentlichkeit?

32 Cf. Anita Guerreau-JalaberT, « Caritas y don en la sociedad medieval occidental », Hispania, 204 (2000), p. 27-62, qui privilégie (à la suite d'Ilana F. SILBER, " Gift-giving in the great traditions. The case of donations to monasteries in the medieval West ", Archives européennes de sociologie, 1995, p. 209-243) le modèle de l'échange généralisé à l'opposé du modèle binaire don/contre-don, dont l'apparente efficacité repose sur la mauvaise compréhension de la notion de mutuus amor (et notre représentation du mutuel comme réciprocité) et sur l'illusion d'optique entretenue par les modalités de conservation des actes de donation (dans les seules archives de l'institution ecclésiastique bénéficiaire - comme si la donation était une pure relation binaire) à l'opposé de la conception de la communion des suffrages dans le cadre de l'ecclesia. La pertinence de ce modèle pour la société médiévale (y compris jusqu'au XVe siècle, comme l'indiquent les travaux de Jacques Chiffoleau, puisque l'introduction d'une mentalité comptable ne remet en aucun cas en cause la pratique des dons pieux, qui constituent une part croissante des revenus d'Église : la mentalité comptable se développe à l'intérieur du schéma de la caritas) n'exclut pas que puisse se rencontrer ici ou là des pratiques que l'on pourrait rattacher à telle ou telle forme d'oblation non caritative (don/contre-don, potlatch, etc.), mais outre que leur sens social effectif échappe certainement à l'examen de formes isolées, il importe de ne pas négliger le rapport hiérarchique et logique entre ces formes et la pratique dominante - sans quoi l'observation de nos pratiques des cadeaux pourrait tout aussi bien nous conduire à nier que la logique sociale dominante dans laquelle nous évoluons est celle des échanges marchands...

33. Émile BENVÉNISTE, Problèmes de linguistique générale, Paris, Gallimard, 1966, p. 227-231.

34. Sur les liens intimes entre le sigillant et le sceau, cf. Brigitte Miriam Bedos-ReZAK, «Medieval Identity: A Sign and a Concept », The American Historical Review, 105 (2000), p. 1489-1533; "Signes d'identité et principes d'altérité au XII siècle : l'individu, c'est l'autre », dans : Bedos-Rezak, IOGNA-PRAT (dir.), L'individu..., p. 43-57 et, pour la Franconie de la fin du Moyen Âge, J.M., «La construction sociale des identités dans l'aristocratie franconienne aux XIVe et XVe siècles : individuation ou identification?", ibidem, p. 79-99.
} 
évocation transparente, c'est-à-dire vecteur de l'émetteur. Toutes choses qui fonctionnent remarquablement bien à propos des rapports entre dieu, l'Église et les chrétiens. La double dissociation établie par la charte entre émetteur et dépositaire du document et entre récepteur du document et du message me parait en effet incroyablement homologique à celle qui concerne les Écritures, le message biblique. On a en effet affaire à un émetteur [dieu], un dépositaire [le clergé] et un destinataire du message [les chrétiens, lors des cérémonies au cours desquelles la parole divine est dite, lue et montrée].

Si une telle homologie n'est pas un hasard (et il serait étonnant qu'il en fût ainsi), on comprend dès lors pourquoi Geoffrey Koziol a pu concevoir les diplômes royaux comme des sortes de prières et pourquoi Brigitte Bedos-Rezak peut voir le sceau fonctionner de manière homologique à l'incarnation du Christ... Mais on comprend aussi l'importance de l'absence de réponse à la charte : si l'émission d'une charte est conçue de manière homologique (ce qui ne signifie évidemment pas : équivalente) à l'émission de la parole divine, la non-réponse du public est à la charte ce que la non-réponse des chrétiens est à la bible 35 .

Si la 'charte' ne prévoit pas de réponse, à l'exact inverse de la 'lettre', ce serait donc moins pour des raisons pratiques (la difficulté voire l'impossibilité pour le public [3] de réagir face à l'émetteur [1]) que pour des raisons essentielles, inhérentes à la nature du document, bref une propriété de la 'charte'. De même qu'il ne s'agit pas d'un document que l'on peut conserver mais d'un document émis pour être conservé, il ne s'agit pas d'un document auquel on ne peut pas répondre mais d'un document auquel on ne doit pas répondre, pas plus qu'on ne doit prétendre répondre à la bible.

La domination symbolique par la non-réponse constitue donc une hypothèse remarquablement économique, puisqu'elle articule aisément des sphères a priori séparées du fonctionnement social. Cette hypothèse laisse cependant ouverte l'alternative suivante : serait-ce en mettant symboliquement en absence les destinataires face aux émetteurs que la 'charte' exclurait symboliquement toute possibilité de réponse? Ou ne s'agirait-il là que d'une grammaire par laquelle la 'charte' traduirait des rapports sociaux de domination avérés - la non-réponse n'étant que le signe (et pas le moyen autrement que de cette manière formalisante) de la domination sociale?

\section{IV.- Faire répondre}

Toutefois, on doit se garder de considérer que tout échange où il y a réponse est une communication hors du cadre d'une domination. Car qu'est-ce qu'une réponse ? Afin de préciser cette question, je vais examiner un autre cas de communication, dans la même région et à la même époque, dans laquelle il y a réponse et qui pourtant s'avère être un indubitable rapport de domination seigneuriale : la Weisung 36 . La Weisung est l'opération au cours de laquelle est publiquement énoncé un ensemble de droits seigneuriaux dans un endroit donné, en général au cours d’un plaid. L’aveu des droits locaux du seigneur était un rituel répété

\footnotetext{
35 Le fait que les chartes aient pu faire l'objet de copies dans des cartulaires, cartulaires dont la fonction sociale reste peu claire puisque les copies n'ont pas la valeur des chartes en justice (et l'alternative des pancartes montre en effet que la forme 'cartulaire' n’avait pas de nécessité absolue...) n’est-il pas déterminé par la situation d'homologie charte/bible ? Le cartulaire est en effet d'une part le moyen de transformer formellement des chartes en un codex, qui est la forme essentielle de la bible. Par ailleurs, la bible est en effet copiée et recopiée en permanence, signifiant que le contenu est indépendant du support (seule l'adéquation du texte au texte étant importante) : la reproduction de chartes dans des cartulaires n'est-elle pas un moyen de montrer que le support et le contenu sont dissociables? L'apparition des instruments notariés correspondrait alors à un changement de finalité des documents et non pas à une modernisation des techniques. Par ailleurs, concernant le rapport entre diplomatique et christianisme, B. FRAENKEL, «L'auteur... » attire l'attention sur la proximité qui existe entre ce qu'elle appelle (p. 418) le «modèle de l'auteur à deux têtes» de la diplomatique (l'auteur nominal qui dit « je »/l'auteur pratique : le scribe) et le «dispositif à deux auteurs » de la « parole opérative » (sacrements et proférations, qui associent celui qui profère et celui sous l'autorité de qui est faite cette profération, dieu ou diable). Si l'on tient compte de ceci, il faudrait alors sans doute modifier le schéma ternaire de la manière suivante :
}
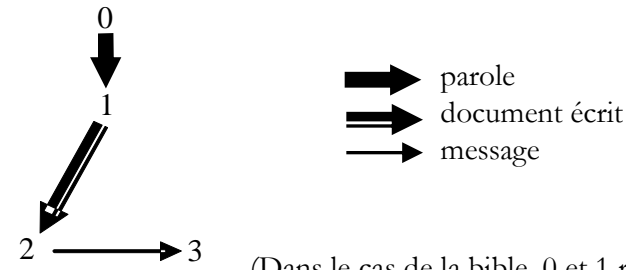

0 origine du message ( je $»)$, faire-écrire et faire-lire

1 origine de l'écrit

2 destinataire de l'écrit et faire-lire

3 destinataire du message

Pères de l'Église d'autre part).

${ }^{36}$ Ce qui suit reprend pour l'essentiel J.M., «Quand faire dire, c'est dire. Le seigneur, le village et la Weisung en Franconie du XIII au XVe siècle », dans : BOUDREAU... (dir.), Information et société..., p. 309-326. 
chaque année. Toutefois, on ne nous dit jamais que les échevins «rappellent» les droits, mais qu'ils les « disent » (sagen, sprechen), « révèlent 》 (offnen ou offenbaren), « communiquent 》 (teilen) et surtout weisen, donc comme si c'était à chaque fois une nouvelle fois... D'ailleurs, l'examen que j'ai eu l'occasion de faire de cette pratique m'a conduit à considérer que ce qui importe est moins le contenu de la Weisung que la Weisung elle-même ${ }^{37}$.

Or celle-ci se déroule essentiellement sous la forme d'une série de questions-réponses. On considère que la forme dominante a été celle d'une succession de couples question-réponse, clairement attestée dans de nombreux documents. D’une manière générale, en effet, le représentant local du seigneur présent, en général l'écoutète (schulthei $\beta$ ), interroge (interrogare, quaere, fragen) les échevins sur les droits du seigneur et ce, même lorsque le seigneur est présent ! Le problème n'est alors pas de savoir si les paysans avaient ou non une certaine latitude pour naviguer/oublier/différer, etc. ${ }^{38}$ Le problème est bien plutôt la signification sociale de l'interaction question/réponse. Il ne s'agit de fait en aucun cas d'une interaction langagière neutre, car elle établit un rapport de forces, bien identifié par les pragmaticiens comme Oswald Ducrot ${ }^{39}$ : la question est une énonciation caractérisée par le pouvoir de mettre l'auditeur dans l'obligation de répondre - pouvoir dont l'efficace a été précisée en particulier par la sociologie des pratiques langagières autour de P. Bourdieu ${ }^{40}$ :

« la question, bien qu'elle se présente comme une demande d'information, est aussi une prise effectuée sur un autre sujet parlant qu'elle constitue, quoi qu'il fasse, en répondeur virtuel - ne serait-ce que pour établir qu'il refuse de répondre. Elle est une main-mise d'ordre symbolique sur le corps, le temps et la parole de l'autre, du simple fait qu'elle brise un silence et ouvre un espace verbal. »

Cet espace verbal est inéluctablement un espace de domination symbolique, déterminé par les conditions pratiques et sociales de l'énonciation. L'interaction question/réponse est par conséquent une pratique essentielle dans laquelle c'est moins le contenu de l'information qui compte que le devoir de donner l'information. Ceci explique notamment le maintien de la procédure de questionnement même lorsque la médiation du langage est modifiée par le recours à un document écrit : en effet, on voit apparaittre, au plus tard au XIVe siècle, une pratique qui consiste à faire lire devant les échevins et/ou les habitants un document énumérant les droits et devoirs du seigneur dans le lieu concerné et à demander aux échevins, à la fin de la lecture ou après chaque clause, s'il en va bien ainsi.

On a donc affaire à un entrelacement de l'écrit et de l'oral, qui montre que la mise par écrit ne signifie en aucun cas la disparition de la prestation orale des échevins. Cette mise par écrit qui ne dépossède pas vraiment la communauté de sa participation dépasse donc clairement le simple problème de la mémoire que, pourtant, les documents invoquent parfois pour justifier le recours à l'écriture ${ }^{41}$. Ce qui me semble

\footnotetext{
37. On observera que tous ces verbes (sprechen, teilen, offnen, offenbaren) rapprochent implicitement la Weisung de la diffusion de la parole divine, car sprechen renvoie notamment aux "paroles» du Christ, offnen/offenbaren à la Révélation (Offenbarung) et teilen à la communicatio, c'est-à-dire la communion... Et l'on notera également que l'un des sens médiévaux de wîsunge est «infusion du saint Esprit ».

38. Plus exactement, les marges de manœuvre des villageois ne sont pas intéressantes en tant que telles, mais en tant qu'elles documentent des situations-limites à travers lesquelles la logique du système apparait sous un autre éclairage. Les louvoiements villageois d'après les Weistümer constituent précisément l'un des principaux objets de Gadi ALGAZI, Herrengewalt und Gewalt der Herren im späten Mittelalter. Herrschaft, Gegenseitigkeit und Sprachgebrauch, Francfort/New York, Campus, 1996.

39. Dire et ne pas dire, Paris, Hermann, 1972 ; Les mots du discours, Paris, Minuit, 1980.

40. Pierre EnCRevé, Michel DE Fornel, «Le sens en pratique. Construction de la référence et structure sociale de l'interaction dans le couple question/réponse », Actes de la Recherche en Sciences Sociales, 46 (1983), p. 7-8.

${ }^{41}$ Le problème n'est pas celui de la confiscation de la parole paysanne par les seigneurs dans le cadre d'un passage linéaire de la mémoire orale à la mémoire écrite, comme s'il y avait eu à l'origine une communication paysanne libre (et orale) qui serait ensuite tombée sous la coupe seigneuriale (et dont le contrôle aurait été assuré par sa mise par écrit, conçue comme une dépossession) schéma de rédaction dépossédante qui sous-tend p ex. le travail de Michael Prosser, Spätmittelalterliche ländliche Rechtsaufzeichnungen am Oberrhein zwischen Gedächtniskultur und Scbriftlichkeit. Untersucbungen am Übergang von analphabetischen zu skeriptualen Überlieferungsformen im Blickfeld rechtlicher Volkskunde, Wurtzbourg, Bayerische Blätter für Volkskunde (Veröff. z. Volkskunde u. Kulturgesch., 47), 1991. Car - et c'est là qu'apparaît tout l'intérêt d'examiner les Weistümer sous l'angle des rapports de communication - l'examen détaillé par Simon TEUSCHER, "Kompilation und Mündlichkeit. Herrschaftskultur und Gebrauch von Weistümern im Raum Zürich (14.-15. Jahrhundert)», Historische Zeitscbrift, 28 (2001), p. 289-333, fait apparaittre que le contenu des Weistümer qu'il étudie résulte de compilations de passages provenant de chartes, censiers et codifications antérieures, parfois vulgarisés (non pour les rendre lisibles par tous mais pour suggérer leur origine populaire) et concernant souvent des seigneurs différents - compilation mise en œuvre dans un cadre désormais villageois (où pouvaient être présents plusieurs seigneurs), à l'occasion de la Weisung. On a donc affaire non pas au détournement seigneurial d'une forme de communication originelle mais à la production d'une nouvelle forme de communication, liée à la formation des communautés villageoises - mais (et c'est là ce qui nous importe ici) une forme de communication qui, pour affirmer la domination seigneuriale, recourt au principe des questions et réponses! En d'autres termes :
} 
par conséquent significatif ici, c'est le maintien, même ténu, de la réponse des échevins : ce ne sont plus eux qui fournissent des données concrètes et précises, ils se contentent de répondre par oui (ou par non) aux questions posées, ce qui montre que l'essentiel est qu'ils répondent.

\section{Conclusion}

Si nous reprenons le problème qui nous intéresse ici, on voit donc bien que la seule présence d'une réponse ne suffit pas pour faire d'une communication un ferment d'espace public. C'est pourquoi j'avais parlé plus tôt de droit de réponse, qui s'oppose tout autant au devoir de réponse (par exemple dans le cadre d'un interrogatoire ${ }^{42}$ ) qu'à l'impossibilité de la réponse. La genèse de la possibilité de réponse, ou l'affaiblissement des modalités de blocage de la réponse, sont des facteurs essentiels de la genèse d'un espace public. D'une certaine manière, on peut considérer que le rôle-clé de la réponse est suggéré par une expression comme celle du « dialogue » entre le roi et la nation, par exemple sous la forme de la consultation des états, bien que les modalités de la réponse soient rarement envisagées et que la notion de « dialogue » soit souvent utilisée de façon métaphorique ${ }^{43}$.

Le principe actif de ce «dialogue » est dans le «droit de réponse » plus que dans la représentativité des états et surtout que dans la possibilité offerte de s'exprimer. Le "droit d'expression» ne fonde pas «l'espace public », c'est le «droit de réponse », qui s'oppose tant au monologue du pouvoir qu'au devoir de répondre des dominés. On domine ainsi moins en parlant qu'en parlant en sorte que l'autre ne puisse pas répondre, soit techniquement (lecture individuelle ${ }^{44}$, audiovisuel), soit par l'intimidation (menace directe ou posture sociale). C'est la raison pour laquelle on devrait sans doute considérer que le véritable enjeu de l'espace public, c'est la possibilité de répondre.

on observe bien ici comment la mise en place d'une domination seigneuriale sur une communauté villageoise est corrélative de la mise en place d'un système de questions et réponses, c'est-à-dire d'une réponse encadrée...

42 Sur la demande de réponse en tant que fondatrice d'un rapport inégal, cf. encore Michael TocH, «Asking the Way and Telling the Law : Speech in Medieval Germany », The Journal of Interdisciplinary History, 16/4 (1986), p. 667-682 ; Gadi AlgazI, "Tradition als Gespräch der Ungleichen. Bauern und Herren un der spätmittelalterlichen Dorfversammlung », dans : Stefan EsDERS, Thomas SCHARFF (dir.), Eid und Wabrheitssuche. Studien zu rechtlichen Befragungspraktiken in Mittelalter und früber Nenzeit, Francfort s/M., P. Lang, 1999, p. 191-210. La signification sociale différente du fait de répondre à une demande de réponse (puisque l'on a dit que les 'lettres' elles-mêmes s'achèvent par une demande de réponse) ne se ramène sans doute pas (ou pas seulement) à la différence entre 'prier' et 'demander', ni sans doute au ton du questionnement (difficile à reconstruire - sans parler que même lorsque le seigneur est présent, ce n'est pas lui qui questionne mais son représentant, issu de la communauté elle-même) : est-ce dû au fait que dans un cas (p. ex. le Weistum), la réponse se trouve dans un document émis par le questionneur lui-même tandis que dans l'autre (la 'lettre') la réponse se trouve dans un document émis par le répondeur lui-même - comme si être émetteur d'un document signifiait être socialement significatif ? Ou est-ce lié à ce à quoi répond la réponse - une demande de réponse ou une question ? On retrouverait alors ici le sens fort qui a pu être attaché à la notion de question, allant jusqu’à la torture... Dans tous les cas, on voit bien qu'il y a réponse et réponse, et que c'est de la nature de celle-ci que dépend la nature de l'échange.

${ }^{43}$ Un exemple entre mille de cet usage métaphorique : Veronika NovÀK, « La source du savoir. Publication officielle et communication informelle à Paris au début du XVe siècle», dans : BOUDREAU... (dir.), Information et société..., p. 154 : «Les rituels comme l'entrée ou le sacre sont conçus comme faisant partie d'un véritable dialogue entre le roi et son peuple où, à côté du message général de paix et de bon gouvernement, certains gestes [ou objets] sont introduits en vue d'une information très concrète, telle l'épée présentée au duc de Bedford... en 1422.»

${ }^{44}$ Tant que les ordres doivent être communiqués oralement (même s'ils sont transmis par écrit, mais destinés à être lus publiquement), les récepteurs peuvent toujours réagir contre l'émissaire représentant le pouvoir - donc contre le pouvoir. C'est clairement le cas avec les crieurs étudiés par Nicolas OfFENSTADT, «Les crieurs publics à la fin du Moyen Âge. Enjeux d'une recherche », dans : BOUDREAU... (dir.), Information et société..., p. 203-217 : on observe bien d'une part sa mise en absence par rapport aux émetteurs, le crieur en tant que représentant étant à la fois présent (corporellement/vocalement) et absent (en tant qu'individualité, d'où par exemple l'usage d'une livrée, etc.), ce qui lui permet de n'apparaitre que comme un relais neutre, un pur représentant - en tant que celui à travers lequel l'émetteur [1] redevient présent. Les crieurs publics apparaissent d'autre part comme les supports d'une interaction - au résultat éminemment variable - construite par l'émission d'une parole qu'on veut sans réponse (information) et un public qui prétend pouvoir répondre à cette parole (communication). On comprend aussi sans doute mieux que les archives nurembergeoises (p. ex. Staatsarchiv Nürnberg, Rep 54a/I, Stadtrechnungsbelege, Nr 1193, 1217) fassent apparâ-tre que les princes et certains aristocrates envoient à la ville non pas des messagers, mais des « souffleurs de trompette », c'est-àdire des crieurs : ceux-ci m’apparaissent comme un moyen de transformer une communication (lettre) en domination symbolique. Dès lors en revanche que se diffuse la norme de la lecture individuelle, il n'y a plus de réaction possible autre qu'arrêter de lire ou brûler le texte... La domination de l'auteur d'un livre sur le lecteur est ouverte en fait largement moins par l'objet-livre en tant que tel que par la lecture individuelle du livre - ce que sous-entend Roland Barthes lorsqu'il s'attaque à notre pratique soumise du texte que nous appelons couramment lecture et/ou littérature : «Notre littérature est marquée par le divorce impitoyable que l'institution littéraire maintient entre le fabricant et l'usager du texte, son propriétaire et son client, son auteur et son lecteur. Ce lecteur est alors plongé dans une sorte d'oisiveté, d'intransitivité... il ne lui reste plus en partage que la pauvre liberté de recevoir ou rejeter le texte : la lecture n'est plus qu'un referendum » (S/Z, Paris, Le Seuil, 1970, p. 10). 
Si l'on corrèle ainsi l'Öffentlichkeit non pas au raisonnement critique (en tant qu'opposé à l'adhésion) mais à la possibilité socialement exercée de la réponse, on est susceptible de dépasser le strict cadre historique défini par Habermas (qui fait de l'Öffentlichkeit une sorte de miracle passager surgi dans un entre-deux de l'histoire européenne), mais aussi d'abandonner la corrélation de l'Öffentlichkeit avec des notions politiques contemporaines (démocratie, public/privé, etc.). L'Öffentlichkeit est susceptible d'être présente partout où une procédure de réponse articulée et collective se met en place - tandis qu'inversement les systèmes de domination sociale se construiraient par l'instauration de formes d'échange sans possibilité de réponse (qui est ce que j’appelle spécifiquement l'information). Ceci modifie radicalement la manière de s'interroger sur la domination par la parole au Moyen Âge, la question qui se pose étant désormais : de quelle manière le pouvoir médiéval instaure-t-il de la non-réponse?

Revenons-en à la raison pour laquelle nous sommes là : la notion d'« espace public » est-elle pertinente, ou heuristique, pour la période médiévale ? Une fois dénoncés l'anachronisme historique de «public » et l'anachronisme théorique d'« espace», comme je l'ai fait initialement, on pourrait estimer qu'il n'y a plus rien à en faire. Par ailleurs, il me semble avoir souligné, à partir d'une analyse de cas médiéval, que la genèse d'un «espace public » conçu comme un espace démocratique de communication concerne moins le problème de la rationalité marchande bourgeoise que le problème de la capacité de réponse : autre limite que je vois à la théorie habermassienne. En revanche, il faut lui savoir gré - et reconnaitre l'intérêt heuristique de son approche, ne serait-ce que par la critique qu'elle suscite - de pousser à l'examen du rapport entre pouvoir et communication, qui va bien au-delà de la seule appropriation des médias par les puissants à laquelle on réduit le plus souvent ce rapport.

À partir de là, j’aurais tendance à considérer plus pertinent - plutôt que de s'accrocher coûte que coûte à la notion d'« espace public» - de construire un système notionnel, fondé par exemple sur la dissociation nette entre information (conçue comme je l'ai dit plus haut comme un échange sans réponse fondateur d'une domination, parce que véridictif) et communication (conçue comme cet échange avec réponse et fondatrice, comme son nom l'indique, d'une communauté - ce qui ne devrait évidemment pas faire accroire qu'il n'y a pas de domination au sein de la communauté, mais elle s'y réalise selon des processus différents), au lieu de l'usage souvent indistinct et indigène (c'est-à-dire en fonction de notre sens commun) qui en est fait aujourd'hui ${ }^{45}$.

\footnotetext{
${ }^{45}$ Le volume mentionné à plusieurs reprises, BOUdREAU... (dir.), Information et société..., est de ce point de vue très significatif. Si certaines communications s'y sont essayées à distinguer les deux ou à préciser le sens de l'une ou l'autre, les deux termes ont été encore trop souvent employés l'un pour l'autre. On observera d'ailleurs qu'entre le colloque - intitulé Informer : institutions et communication (XIII ${ }^{e}-X V^{e}$ siècle) - et la publication des actes, la mention de la communication a inexplicablement disparu du titre - et avec elle l'évocation du rapport entre « communiquer » et « informer».
} 